\title{
Role of Combination Therapy with SGLT2 Inhibitor with Metformin as Initial Treatment for Type2 Diabetes- Advantages of Oral Fixed Drug Pill Like Empagliflozin/ Metformin in Patients with Cardiovascular and Renal Risk-A Short Communication

\author{
Dr. Kulvinder Kochar Kaur, M.D., ${ }^{1 *}$ Dr. Gautam Allahbadia M.D.(Obstt \& Gynae), D.N.B, ${ }^{2}$ \\ Dr. Mandeep Singh M.D.DM.(Std)(Neurology) ${ }^{3}$
} \\ ${ }^{1}$ Scientific Director, Dr. Kulvinder Kaur Centre For Human Reproduction, 721,G.T.B. Nagar, Jalandhar-144001, Punjab, India.
}

${ }^{2}$ Scientific Director, Ex-Rotunda-A Centre for Human reproduction, 672, Kalpak Garden, Perry Cross Road, Near Otter's Club, Bandra (W)-400040, Mumbai, India.

${ }^{3}$ Consultant Neurologist, Swami Satyanand Hospital, Near Nawi Kachehri,Baradri, Ladowali road, Jalandhar, Punjab. India.

kulvinder.dr@gmail.com

*Corresponding Author: Dr. Kulvinder Kochar Kaur, Scientific Director, Dr. Kulvinder Kaur Centre For Human Reproduction, 721,G.T.B. Nagar, Jalandhar-144001, Punjab, India.

\section{Abstract}

The incidence of Type 2 Diabetes is increasing worldwide, associated with obesity, so much so that the term diabesity got coined. Not only is the incidence increasing but both morbidity and mortality are increasing worldwide. Thus it is empirical to treat T2DM efficiently so that the cardiovascular (CV) and renal problems get taken care of. All the ADA/AACE/ACE recommend treating with a combination therapy in patients having a high $\mathrm{Hb} \mathrm{A1c}$. The preferred drugs has been the initial metformin and recent multiple trials and studies have shown the superiority of the sodium glucose cotransporter 2(SGLT2) inhibitor of which empagliflozin along with metformin has been tried in fixed dose combination, whose advantages are not only that they take care of T2DM but have simultaneous weight reducing, blood pressure reducing, increased compliance along with individualization of therapy for the patients having a risk of major adverse CV event as tested by the EMPAREG-OUTCOME in 7020 patients along with further corroborated with more studies. Thus simultaneous control ofT2DM, BP, weight, CV outcomes and renal effects makes a combination of empagliflozin 10 or $25 \mathrm{mg}$ in combination with metformin, both having complementary roles and need to be the initial treatment of choice in a fixed dose pill, making it easier for the patient to need just one tablet. In 2016 FDA has also recommended the use of this for this group of patients having major risk of CV events.

Keywords: T2DM; SGLT2 Inhibitors; metformin; empagliflozin; FDA; CV event

Conventionally a stepwise approach has been used to treat type 2 diabetes mellitus (T2DM), like initial lifestyle interventions and metformin being the first line therapy, followed by addition of 2nd line therapy once optimal glycaemic control is not achieved. Yet, an alternative more intensive approach remains an initial combination therapy. There is a disparity in current guidelines regarding patients glycated haemoglobin
(Hb A1c) for which initial combination therapy should beconsidered as per the American Diabetes Association (ADA) and European Association for the Study of Diabetes(EASD)if $\mathrm{Hb}$ A1cis $>1,5 \%$ above the patient's target or is indicated as per American s Association Of Clinical Endocrinologists (AACE) and the American College of Endocrinology (ACE) if Hb A1 cis > 7.5\%[1,2]. In favour for initial combination therapy instead of 
Role of Combination Therapy with SGLT2 Inhibitor with Metformin as Initial Treatment for Type2 Diabetes-Advantages of Oral Fixed Drug Pill Like Empagliflozin/Metformin in Patients with Cardiovascular and Renal Risk-A Short Communication

metformin monotherapy proponents argue that this avoids clinical inertia along with potential for more pronounced and earlier improvement in glycaemic control, which might $=>$ a legacy effect $[3,4]$. In the UK Prospective Diabetes Study (UKPDS), especially newly diagnosed Type2 diabetes mellitus (T2DM) participants were randomized to intensive therapy achieved a lower $\mathrm{Hb} \mathrm{A} 1 \mathrm{c}$ as compared to participants randomized to conventional therapy $(7,0 \%$ versus 7.9\%)[5]. Once the study ended, this difference in $\mathrm{Hb} \mathrm{A} 1 \mathrm{c}$ between the 2 groups was lost by one year; though after 10yrs of follow up, the intensive therapy group had a lower risk of myocardial infarction along with T2DM related death as compared to conventional therapy[6].

Sodium-glucose cotransporter 2(SGLT2) inhibitors, remain an attractive choice for starting initial combination therapy with metformin. Their mechanism of action is insulin -independent, i.e. by increasing the urinary excretion of glucose[7]. Along with that weight reduction and lowering of blood pressure(BP) occurs in contrast to placebo[7]. Empaglifozin, canaglifozin, and dapaglifozin have been found to decrease the risk of hospitalization from heart failure and stroke in people having T2DM with established cardiovascular disease or multiple cardiovascular risk factors significantly [8-10]. A decrease in risk of total outcome of cardiovascular death, myocardial infarction and stroke in people with T2DM and established atherosclerosis, cardiovascular disease (CVD) with SGLT2 inhibitors use was obvious in these cardiovascular outcome trials [11]. Earlier meta-analysis compared initial combination therapy of various antihyperglycaemic agents with metformin to metformin monotherapy [12]. In this meta-analysis only one SGLT-2 Inhibitor, dapaglifozin got included in the meta-analysis. Further the efficacy measures only included glycaemic control, with effects on bodyweight and BP were not examined. Recently Cai et al [13] conducted a meta-analysis where they examined initial combination therapy which included SGLT2 inhibitor and metformin in treatment naïve T2DM patient. But all treatment groups from each trial of combination SGLT2 inhibitor and metformin were not included, with a discrepancy between number of subjects included in the efficacy and safety assessments. Moreover dose response relationships were not included. Empaglifozin is an SGLT 2 Inhibitor which is in common with other agents in this class and decreases the elevated blood glucose levels by inhibiting SGLT2, that is the main transporter needed for reabsorption of glucose from the glomerular flltrate and hence increases urinary excretion of glucose[14-16]. In contrast although the mechanism of action of metformin is not fully understood, its antihyperglycaemic effects are thought to arise from the suppression of hepatic gluconeogenesis[17]. Since both agents have complementary mechanism of action [18] these 2 drugs have the potential to offer improved glucose control compared with that achieved with individual agents. An oral fixed dose, single pill combination of empaglifozin and metformin is also available for patients having T2DM in a range of dose combinations that may help in individualizing therapy [19]. This approach might decrease the burden of pill for patient and hence $=>$ simplified medical costs as compared to the "loose-dose" combination in patients with T2DM[20]. Ultimately this improved adherence to therapy might ultimately help to achieve better glycaemic control in T2DM[20,21]. Moreover, with increasing evidence that supports initial combination therapy initially for T2DM patient irrespective of the HbA1c levels given in current treatment guidelines[20], or the use of early intensification therapy $[22,23]$, suggests an increasing role for these fixed dose combinations in management of T2DM. In 2016, a new indication for empagliflozin was added by the Food and Drug Administration), namely to decrease the risk of major adverse cardiovascular (CV)events with T2DM in patient and CV disease (CVD) [20], once the EMPAREG OUTCOME(EMPA glozin Removal of Excess Glucose: cardiovascular OUTCOME Event Trial in T2DM Patients) (NCT011131676) got published[24]. EMPA-REG OUTCOME was a randomized, double blind trial which assessed the effect of empagliflozin $110 \mathrm{mg}$ or $25 \mathrm{mg} /$ day) vs placebo, added to standard care, on CV outcomes in 7020 patient with T2DM and existing CVD over a median follow up of 3.1 yrs. Most patient in the empagliflozin group(74\%) were also receiving background metformin. Treatment with empagliflozin was associated with $14 \%$ relative risk reduction (RRR) in CV death, nonfatal myocardial infarction, or nonfatal stroke, 38\% RRR in CV death; and 32\% RRR in death of any cause[25]. RR for hospitalization for 
Role of Combination Therapy with SGLT2 Inhibitor with Metformin as Initial Treatment for Type2 Diabetes-Advantages of Oral Fixed Drug Pill Like Empagliflozin/Metformin in Patients with Cardiovascular and Renal Risk-A Short Communication

heart failure was also decreased by $35 \%[25]$. Basic mechanisms for the $\mathrm{CV}$ benefits of empagliflozin are not understood fully, but changes in arterial stiffness, alterations in cardiac oxygen demand, cardiorenal effects, decrease in albuminuria and uric acid, and the established effects of SGLT2 inhibitors like lowering glucose, weight and BP have been proposed[25]. The EMPA-REG OUTCOME trial also showed microvascular benefits of empagliflozin in the sense they slow down the progression of kidney disease (defined as incidence of worsening nephropathy)vs placebo when added to standard care [26]. Both short and long term benefits, in terms of significant decreases in urinary albumin-to-creatine ratio, in patients with T2DM and established CVD was found on exploring the findings of this trial[27]. These findings are reflected in current guidance for T2DM management issued by ADA[28] and AACA/ACE[29] that refers to the possible benefits of empagliflozin on cardiac and renal outcomes.

Watching the findings of EMPA-REG OUTCOME [25], role of empagliflozin in the treatment was evaluated in a range of treatment combinations and considered for use in earlier stages of T2DM. Since metformin[3033], has also been associated with improvements in $\mathrm{CV}$ outcomes, combination therapy with empagliflozin might also confer CV benefits in patients with DM. Considering the potential benefits of dual therapy with empagliflozin and metformin in patients with T2DM, besides frequent requirement for dual therapy Goldman 2018 reviewed on the efficacy and safety of empagliflozin/metformin combination therapy. They found that combination of empagliflozin/metformin offers the potential to improve glycemic control in T2DM and decreases body weigh patients and BP, vs each agent individually, with a management risk profile. Thus this combination could be suitable for patients with T2DM who are inadequately controlled by metformin, especially in patients who would benefit from modest reductions in BP and body weight who have risk factors for CV diseases or reducing renal function. Further empagliflozin/metformin is also available as a single-pill combination that has the potential to provide a simplified regimen and could =>improved clinical outcomes compared with administration of individual tablets.[34].

Further Milder et al compared the efficacy and safety of i) sodium-glucose cotransporter2 inhibitor combination therapy in treatment naïve T2DM adults, ii) initial high and low dose SGLT2 inhibitor combination therapy. They used randomized controlled trials(RCT's) of initial SGLT2 combination therapy. Mean differences (MD) for changes from baseline (Hb A1c, weight, BP) after 24-26weeks of treatment and relative risks (RR, safety) were calculated using a random effects model. Risk of bias and quality of evidence was assessed. In 4RCT's $(n=3749)$ there was moderate quality of evidence that SGLT2 inhibitor /metformin combination therapy resulted in a greater reduction in $\mathrm{Hb} \mathrm{A} 1 \mathrm{c}(\mathrm{MD}(95 \% \mathrm{CI})$;$0.55 \%(-0.67,-0.43)$ and weight $(-2.00 \mathrm{Kg}(-2.34,-1.66))$ compared with metformin monotherapy, and a greater reduction in $\mathrm{Hb}$ A1c $(-0.59(-0,72,-0.46)$ and weight $(-0.57 \mathrm{~kg}(-.0 .89,-0.25)$ compared with SGLT2 inhibitor monortherapy. The high dose SGLT2 inhibitor / metformin combination resulted in similar $\mathrm{Hb} \mathrm{A1c}$ but more weight reduction ; $(-0.47 \mathrm{~kg}(-0.88,-0.6)$ than the low dose combination therapy. The RR of genital infection with combination therapy was 2.22 (95\%CI$1.46,3.40$ ) compared with metformin and SGLT2 monotherapy. Thus conclusions drawn were initial SGLT2/metformin combination therapy has glycaemic and weight benefits compared with either agent alone and appears safe. High dose SGLT2 inhibitor / metformin combination therapy appears to have modest weight, but no glycaemic benefits compared with the low dose combination therapy[35].

\section{REFERENCES}

[1] Davies MJ, D’Alesso DA, Franklin J,Kernan WN, Mathieu C, MingroneG, Rosing P,et al. Management of Hyperglycemia in Type2 Diabetes 2018.A consensus report by the American Diabetes Association (ADA) and the European Association for the Study of Diabetes(EASD). Diabetes Care 2018;41:2669-2701.

[2] Garber AJ, Abrahamson MJ, Brazilay JL,BlondeL, Bloomgarden ZT, Bush MA, Dagogo-Jack S,etal. Consensus statement by the American Association of clinical Endocrinology on the Comprehensive Type 2 Diabetes Management Algorithm-2018Executive Summary,Endo Pract 2018;24:91-120.

[3] Cersimo E,Johnson EL, Chovanes C, Skolnik N. Initiating therapy in patients newly diagnosed 
Role of Combination Therapy with SGLT2 Inhibitor with Metformin as Initial Treatment for Type2 Diabetes-Advantages of Oral Fixed Drug Pill Like Empagliflozin/Metformin in Patients with Cardiovascular and Renal Risk-A Short Communication

with Type2 Diabetes: Combination therapy vs a stepwise approach. Diabetes Obes Metab 2018;20:497-507.

[4] Cahn A, Cefalu WT. Clinical considerations on use of initial combination therapy in Type2 Diabetes. Diabetes Care 2016;39:S137-S145.

[5] UK Prospective Diabetes Study (UKPDS) Group. Intensive blood glucose control with sulfonoylureas or insulin compared with conventional treatment and risk of complications in patients with Type2 Diabetes.(UKPDS33). Lancet 1998;352:837-853,

[6] Holman RR, Paul SK, Bethel MA, Matthews DR,Neil HA.10year follow up of intensive glucosecontrol in Type2 Diabetes.N Engl J Med 2008;359:15771589.

[7] Ferrannini E.Sodium glucose cotransporters and their inhibition:Clinical Physiology.Cell Metab 2017;26:27-38.

[8] Neal B,Perkovic V, Mahaffey KW,De Zeeuw D, Fulcher G, Erondu N, Shaw W,etal. Canagliflozin and cardiovascular and renal events in Type2 Diabetes N Engl J Med 2017;377: 644-657.

[9] Zinman B, Wanner C, Lachin JM, Filtchett D, Bluhmki E,Hantel S, Mattheus M,etal. Empagliflozin, cardiovascular outcomes, and mortality in Type2 Diabetes. N Engl J Med 2015;373:2117-2128.

[10] Wiviott SD, Raz I,Bonaca MP, Mosenzon O, Kato ET, Cahn A, SilvermanMG, Zelniker TA,etal. Dapaglofozin and cardiovascular outcomes in Type2 Diabetes . N Engl J Med 2018.

[11] Zelniker TA, Wiviott SD, Raz I, Im K,Goodrich EL, Bonaca MP, Mosenzon O, Kato ET,etal. SGLT2 inhibitors for primary and secondary prevention of cardiovascular outcomes in Type2 Diabetes:a systematic review and metaanalysis of cardiovascular outcome trial. Lancet 2018;393:31-39.

[12] Phung OJ, Sobieraj DM, Engel SS, Rajpathak SN. Early combination therapy for treatment of Type2 Diabetes: systematic review and metaanalysis. Diabetes Obes Metab 2014;16:410417.
[13] Cai X, Gao X, Yang W, Han X, Ji L. Efficacy ansd safety of initial combination therapy in treatment -naïve Type2 Diabetespatients: a systematic review and meta-analysis.Diabetes Ther 2018;9:1995-2014.

[14] De Fronzo RA, Davidson JA, Del PratoS, The role of kidneys in glucose homeostasis-a new pathtowards normalizing glycaemia. Diabetes Obes Metab 2012;14:5-14.

[15] Ferrannini E., Muscelli E, Frascerra S,etal. Metabolic response to sodium glucose cotransporter 2 inhibition in Type2 Diabetic patients.J Clin Invest 2014;124:499-508.

[16] BoehringerIngelheim Pharmaceuticals Inc. SYNJARDY (empagliflozin and metformin hydrochloride) tablets for oral use prescribing information.http://docs.boehringer-ingelheim. com/Prescribing\%20 Information/PIs/ Synjardy/Synjardy.pdf. Published December 2017.Accessed May 25,2018.

[17] An H,He I.Current understanding of metformin effects in control of hyperglycemia in diabetes.J Endocrinol 2016;228:R97-R106.

[18] Macha S, Dietrich S, Mattheus M, Sernan LJ, BroedlUC, Woerle HJ. Pharmacokinetics of empagliflozin, a sodium glucose cotransporter 2(SGLT2) inhibitor, and metformin following coadministration in healthy volunteers.Int J Clin Pharmacol Ther 2013;51:132-40.

[19] Rojas C, Link JMeinicke T, Macha S. Pharmacokinetics of fixed dose combinations of empagliflozin /metformin comparedwith individualtablets in healthy subjects. Int J Clin Pharmacol Ther 2016;54:282-92.

[20] Hutchins V,Zhang B,Fleurence RL, Krishnarajah G, Graham J.A systematic review of adherence treatment satisfaction and costs ,in fixed dose combination regimens in Type2 Diabetes. Curr Med Res Opin 2011;27:1157-1168.

[21] Benford M, Milligan G,Pike J ,Anderson P, Piercy J, Fermer S. Fixed dose combination antidiabetic therapy: real world factors associated with prescribing choices and relationship with patient satisfaction and compliance. Adv Ther 2012;29:26-40. 
Role of Combination Therapy with SGLT2 Inhibitor with Metformin as Initial Treatment for Type2 Diabetes-Advantages of Oral Fixed Drug Pill Like Empagliflozin/Metformin in Patients with Cardiovascular and Renal Risk-A Short Communication

[22] Bianchi C, Damele G, Dardano A, Miccoli R, Del PratoS. Early combination therapy with oral glucose lowering agents in Type2 Diabetes. Drugs 2017;77:247-264.

[23] Cahn A, Cefalu WT. Clinical considerations for use of initial combination therapy in Type2 Diabetes. Diabetes Care 2016;39:S137-S145,

[24] US Food and Drug Administration; FDA News releas: FDA approves Jard-iance to reduce cardiovascular death in adults with Type2 Diabetes. http://www.fda,gov/news events/ newsroom/press announcements/ucm311517. html. Published 2016.Accessed march 8,2017.

[25] Zimman B, Wanner C, Lachim JM,etal. Empagliflozin, cardiovascular outcomes and mortality in Type2 Diabetes. N Engl J Med 2015;373:2117-2128.

[26] 26. Wanner C, Lachim JM ,et al. Empagliflozin and progression of kidney disease in patients withType2 Diabetes. N Engl J Med 2016;375:324334.

[27] Cherney DZI, Zimman B, Inzucchi SE, etal. Effect of empagliflozin on the urinary-albumin-tocreatinine ratio in patients with Type2 Diabetes and established cardiovascular disease: an exporatory analysis from the EMPA-REG OUTCOME:randomized placebo control trial. Lancet Diabetes Endocrinol 2017;5:610-621.

[28] American Diabetes Association .9.Cardiovascular disease and risk management standards of medical care in diabetes-2018.Diabetes Care 2018;41:S86-S104.
[29] Garber AJ, Abrahamson MJ, Barzilay JL, etal. ConsensusstatementbytheAmerican Association of Clinical Endocrinologists and American College of Endocrinology on the comprehensive Type2 Diabetes management algorithm-2017 executive summary. Endocr Pract 2017;23:207-238.

[30] UK Prospective Diabetes Study(UKPDS) Group. Effect of intensive blood glucose control in with metforminon complications in overweight patients with Type2 Diabetes(UKPDS 34).Lancet 1998;352-854-865.

[31] Selvin F, Bolen S, Yeh HC,et al. Cardiovascular in trials in outcomes of oral antidiabetes medication Type2 Diabetes: a systematic review. Arch Int Med 2008;168:2070-2080.

[32] El-Messoudi S,Rongen GA, Riksen NP. Metformin therapy in diabetes: role in cardio protection. Curr Atherosclerosis Rep 2013:15:314.

[33] Holden SE, Jenkins-Jones S, Currie CJ. Association between insulin monotherapy versus insulin plus metformin and the risk of cause mortality and other serious risk outcomes:a retrospective cohort study. PLoSOne 2016;11:e0153594.

[34] Goldman JD. Combination of empagliflozin and metformin therapy: A consideration of its place in Type2 Diabetes Therapy. Clinical Medical insights Endocrinology and Diabetes 2018;11:1-11

[35] Miller TY, Stocker SL, Shaheed CA, McGrath -Cadell L, Samocha-Bonet D, Greenfield JR, Day RO. Combination therapy with an SGLT2 inhibitor as Initial Treatment for Type2 Diabetes: a systematic review and meta-analysis.J Clin Med 2019;8:45.

Citation: Dr. Kulvinder Kochar Kaur, Dr. Gautam Allahbadia, Dr. Mandeep Singh. Role of Combination Therapy with SGLT2 Inhibitor with Metformin as Initial Treatment for Type2 Diabetes-Advantages of Oral Fixed Drug Pill Like Empagliflozin/Metformin in Patients with Cardiovascular and Renal Risk-A Short Communication. Archives of Diabetes and Endocrine System. 2019; 2(1): 15-19.

Copyright: (C) 2019 Dr. Kulvinder Kochar Kaur, Dr. Gautam Allahbadia, Dr. Mandeep Singh. This is an open access article distributed under the Creative Commons Attribution License, which permits unrestricted use, distribution, and reproduction in any medium, provided the original work is properly cited. 